\title{
Acute Necrotising Encephalitis In Young Adult Female: A Rare Experience
}

\author{
Dr.Dilip Roy, Dr.Subhadeep Banerjee, Dr.Tanmoy Pal, Dr. Aindril Bhowmic \\ (Department Of General Medicine,Medical College Hospital,Kolkata,India) \\ (Department Of General Medicine,Medical College Hospital,Kolkata,India) \\ (Department Of General Medicine,Medical College Hospital,Kolkata,India) \\ (Department Of General Medicine,Medical College Hospital,Kolkata,India)
}

\begin{abstract}
Acute necrotizing encephalopathy (ANEC) is a rare disease well recognized in Japan but few cases have been reported from Indian subcontinent. Many cases of ANEC have been reported in the pediatric literature, but very few cases have been described in adults. We describe here a case of adult onset ANEC with the neuro-imaging findings.
\end{abstract}

Key Words: Bilateral Symmetric Brain Involvement, Post-Viral

\section{Introduction}

Acute necrotizing encephalopathy (ANEC) of childhood is a rare disease first described in Japan by Mizuguchi and Colleagues in 1995. The disease has also been reported from the Far East.To our knowledge, two cases had been reported from India-one from AIIMS,Delhi and other from Pune Medical College, Maharastra.Our patient, a $19 \mathrm{yr}$ female admitted with altered sensorium, involuntary movements and rigidity of limbs with a preceding history of fever 3 weeks back. This young female rapidly deteriorated and succumbed to death within few days. ANEC was diagnosed on the basis of clinical features and neuro-imaging findings.

\section{Case report}

2.1 A $19 \mathrm{yr}$ old female, mother of a 3 months old baby, admitted in our hospital with altered sensorium for 20 days and involuntary movements of all four limbs for 10 days, which was preceded by fever 3 wks back . Fever was associated with headache, cough and rhinorrhea. The patient had no antecedent diarrhoea, conjunctivitis, arthralgia, or rash. There was no history of any dog bite, recent travel, vaccinations, HSV/VZV infection, hemorrhagic disease, ear discharge and head injury. Patient was initially treated in a private hospital with inj. Acyclovir, inj. Ceftriaxone, tab-Levetiracitam, but with little improvement. On admission, her Pulse82/min, R/R-18/min, BP-130/84, no organomegaly or lymphadenopathy.CNS examination revealed- initial GCS E4V2M5(which worsened to E1V1M2 in a short interval), pupillary reflex present, corneal reflex present, doll's eyeball phenomenon absent . Fundoscopy was normal. 3rd, 4th, and 6th cranial nerves examination was grossly normal. Both extensor and flexor tones were increased around the elbow joint, flexor tone was increased around wrist and the interphalangeal joints. Increased extensor tone in both lower limbs except around Rt. knee joint where flexor tone was increased. DTR in upper limbs -normal, in lower limbs-brisk, plantar B/L-extensor. Ankle clonus present. Dystonic tremor present in all 4 limbs. Other cranial nerves, power, gait, and sensory system could not be tested for want of cooperation. Other physical examinations were normal.

2.2 Investigations show-CH- Hb\%-10.9,TC-7800,DC-N66 L30 E4 PL-1.6L,ESR-16.KFT \& LFT-normal,FBS96,Na/K-141/2.6,Ca-8.2,Urine RE/ME,CXR \& USG whole abdomen-normal, serology for JE,HSV,HIV1\&2,HBV and HCV were normal. Widal \& MPDA were negative. CSF study revealed cell-2/cc, all mononuclear, sugar- $82 \mathrm{mg} / \mathrm{dl}$, protein- $342 \mathrm{mg} / \mathrm{dl}$, ADA- 4.6

Plain CT scan of brain showed bilateral symmetrical hypodense lesions involving basal ganglia, thalami, and extending into the fronto-parietal region.(Fig:1)

Plain MRI of brain showed bilateral symmetrical lesions in basal ganglia, caudate nucleus and lateral part of thalami which are hypointense in T1 and hyperintense in T2 \& T2 FLAIR.. There were also patchy hemorrhagic component in b/l basal ganglia.(Fig:2) 


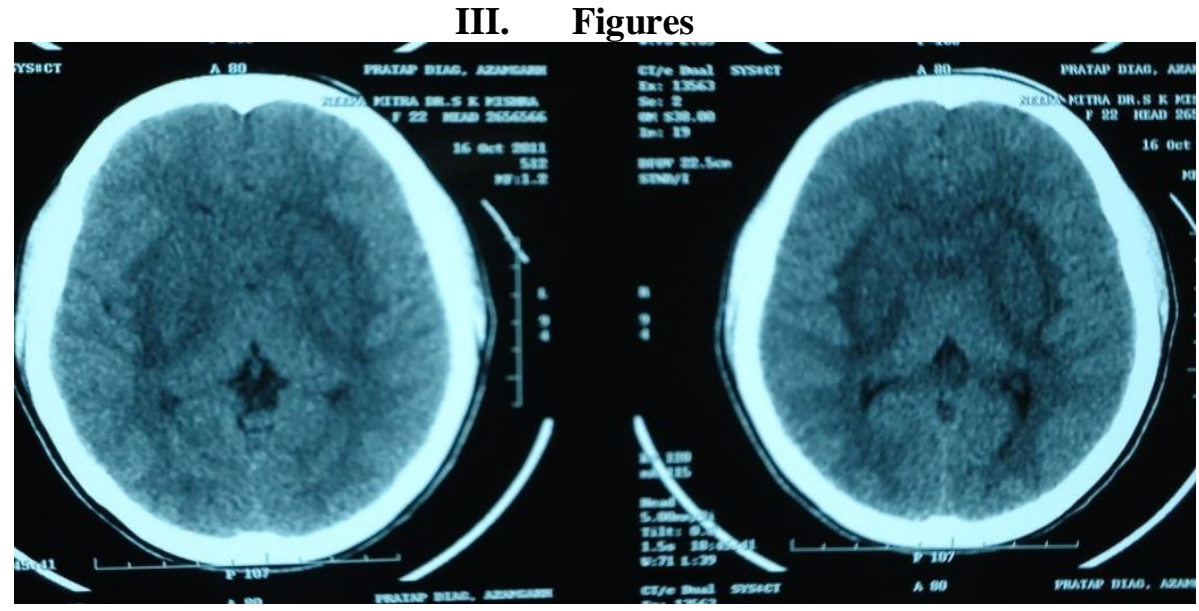

fig:1- bilateral symmetrical ill-defined hypodensity involving both basal ganglia and extending in both frontotemporal and thalamic regions.

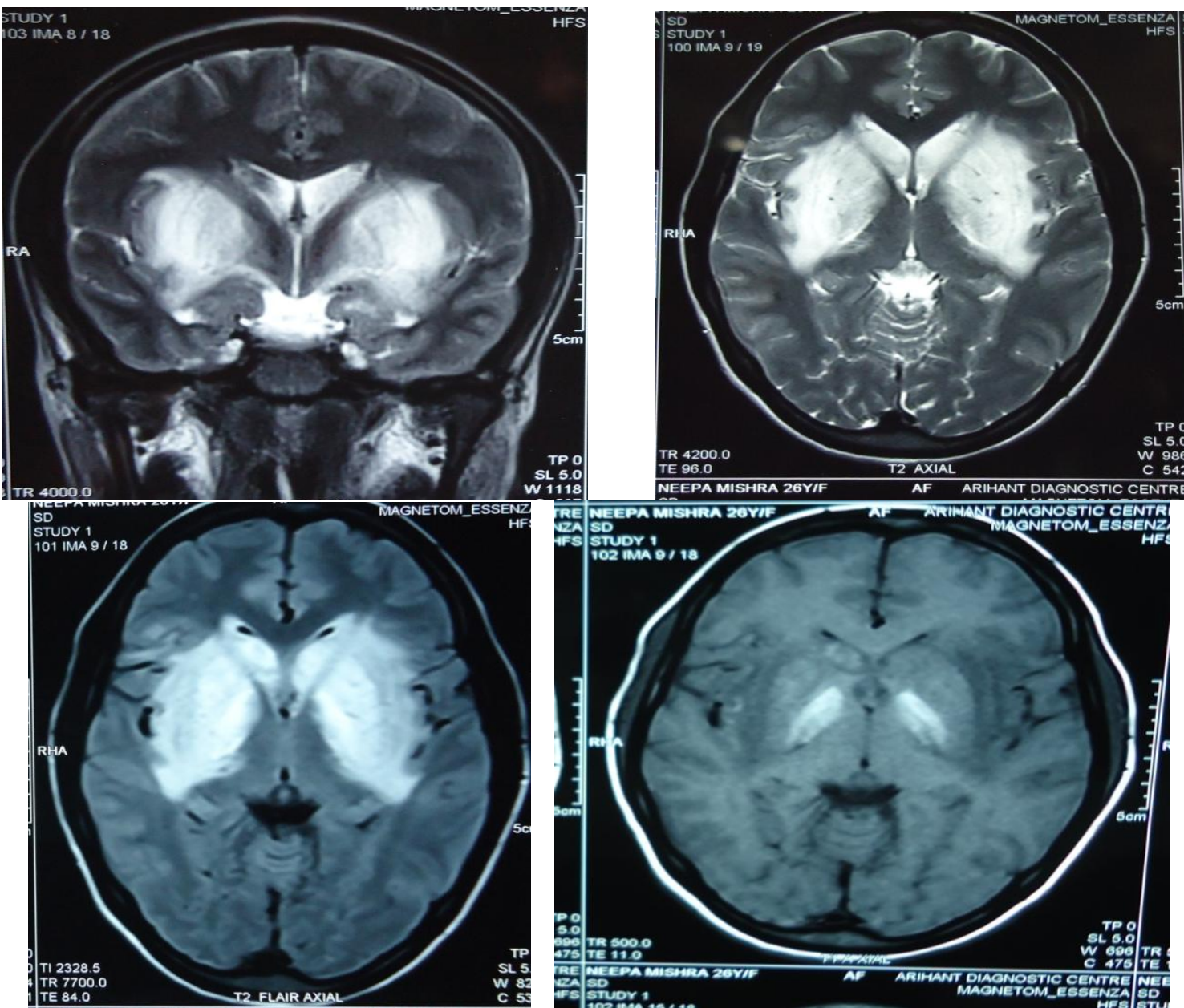

fig:2-Symmetric hyperintensity in b/ basal ganglia, caudate nucleus \& lateral part of thalami on T2 weighted images with patchy hemorrhagic component in b/l basal ganglia. The lesions are also hyperintense in T2 FLAIR \& Tl images.

Based on clinical symptoms, CSF features and MRI findings, a diagnosis of acute necrotizing encephalopathy was made.

Patient was treated with inj. acyclovir, inj. Ceftriaxone (2gm) for $2 \mathrm{wks}$, inj.methylprednisolone $1 \mathrm{gm}$ for 3 days followed by dexamethasone $24 \mathrm{mg}$ in divided doses, tab. trihexyphenidyl $2 \mathrm{mg}$ tid, tab baclofen $10 \mathrm{mg}$ bid, tab clonazepam $2 \mathrm{mg}$ bid, physical rehabilitation and other supportive management. Unfortunately she expired after 20 days of admission. 


\section{Discussion}

Acute necrotizing encephalopathy (ANEC) is a clinical and neuroradiologic entity. The etiology of ANEC is unknown. Influenza A virus, mycoplasma, herpes simplex virus and human herpes virus-6 have been reported as common causative agents, directly or through an immune-mediated mechanism. ${ }^{2-4}$ It is believed that some virus or its variant causes rapid development of intracranial cytokine formation which causes blood brain barrier damage in particular regions of brain resulting in localized oedema, congestion and hemorrhage, without any signs of direct viral invasion or post infectious demyelination. ${ }^{5-7} 24 \%$ of reported cases are related with influenza associated infection. ${ }^{1,2}$ Certain symptomatic medications used in the early stage of viral infection might play a role in ANEC--- like salicylates in Reye's syndrome. The disease affects young children of both sexes but in adults it is very rare. This disease manifests as acute encephalopathy following 2 to 4 days of fever and minor symptoms of the respiratory and/or gastrointestinal systems. ${ }^{1}$ The hallmark of this encephalopathy consists of multifocal, symmetric brain lesions affecting the bilateral thalami, and/or cerebral periventricular white matter, brainstem tegmentum, or cerebellar medulla. ${ }^{8}$ According to Mizuguchi et al, the localized edema, congestion or hemorrhage were more severe in the center of thalamus than periphery producing the concentric appearance on imaging. ${ }^{8}$ Some diseases, such as Reye's syndrome, vascular occlusion, tumor, hemorrhage of the thalamus, Sandhoff disease, or Leigh and Wernicke encephalopathies must be differentiated clinically, radiologically, or pathologically. ${ }^{1}$ In our patient, there was no suggestion of these diagnoses either clinically or radiologically. The prognosis is usually poor. Focal neurologic deficits are common sequelae. ${ }^{1,2}$ The presence of hemorrhage and localized tissue loss on MRI may suggest a poor prognosis. Okumura $e t \mathrm{al}^{10}$ concluded that administration of steroid within 24 hours after the onset was related to better outcome of children with ANEC without brainstem lesions. So, steroid therapy was tried in our patient.

\section{Conclusion}

Acute Necrotizing Encephalopathy is a rare disease with unknown etiology which resembles viral encephalitis albeit with a worse prognosis.

\section{References}

[1]. Mizuguchi M, Abe J, Mikkaichi K et al. Acute necrotising encephalopathy of childhood; A new syndrome presenting with multifocal symmetric brain lesions. J Neurol Neurosurg Psychiatry 1995; 58: 555-561.

[2]. Mizuguchi M. Acute necrotising encephalopathy of childhood; A novel form of acute encephalopathy prevalent in Japan and Taiwan. Brain Dev 1997; 19: 81-92.

[3]. Wang HS, Huang SC. Acute necrotizing encephalopathy of childhood. Chang Gung Med J 2001; 24: 1-10

[4]. Barkovich AJ. Toxic and metabolic brain disorders. In : editors Pediatric Neuroimaging, 4th ed Philadelphia: Lippincott Williams and Wilkins; 2005: 76-189

[5]. Sugaya N. Influenza associated encephalopathy in Japan: pathogenesis and treatment. Pediatr Intl 2000; 42: 215-218.

[6]. Yoshikawa H, Yamazaki S, Watanabe T, Abe T. Study of influenza associated encephalopathy in children during the 1997 to 2001 influenza season. J Child Neurol 2001; 16: 885- 890.

[7]. Morishima T, Togashi T, Yokota S et al. Encephalitis and encephalopathy associated with an influenza epidemic in Japan. Clin Inf Dis 2002; 35: 512-517.

[8]. Mizuguchi M, Hayashi M, Nakano I et al. Concentric structure of thalamic lesion in acute necrotizing encephalopathy. Neuroradiology 2002; 44: 489-493.

[9]. Kim JH, Kim IO, Lim MK et al. Acute necrotizing encephalopathy in Korean infants and children: imaging findings and diverse clinical outcome. Korean J Radiol 2004; 5: 171-77.

[10]. Okumura A, Mizuguchi M, Kidokoro $\mathrm{H}$ et al. Outcome of acute necrotizing encephalopathy in relation to treatment with corticosteroids and gammaglobulin. Brain Dev 2008; May 2. [Epub ahead of print] 